\title{
Strong Anticipation: Complexity Matching in Interpersonal Coordination
}

\author{
Vivien Marmelat*† \\ Didier Delignières* \\ Peter Beek $\dagger$ \\ (*) EA 2991 Movement to Health, Montpellier-1 University, France \\ (†) MOVE, VU University Amsterdam, The Netherlands \\ E-mail:vivien.marmelat@univ-montp1.fr
}

\begin{abstract}
Anticipation in sensori-motor synchronization tasks is often attributed to the presence of internal model based on situation regularities. However this interpretation seems unable to explain the synchronization of an organism with a complex environment. Instead the notion of "strong anticipation" provides a new point of view about synchronization between complex systems. It implies a global coordination on non-local time scales between an organism and its environment. The matching between the long-range correlations of the organism and the long-range correlations of the environment could attest for the presence of such a strong anticipatory process. We propose here to test the presence of strong anticipation in interpersonal coordination task. Results show a close matching of fractal exponents of participants within a dyad. Moreover this matching cannot be attributed to only short-term adaptations as revealed by the low percentage of local cross-correlations.
\end{abstract}

\section{Introduction}

\subsection{On strong anticipation}

Sensori-motor synchronization (SMS), i.e. synchronization between an organism and its environment, is often associated with anticipation processes. For instance in tapping task with auditory periodic metronome, a negative mean asynchrony is frequently observed, suggesting that participants tend to anticipate rather than to react to the occurrence of the stimulus [1]. This kind of anticipatory behavior has been widely interpreted as the consequence of the construction of internal models by the participants. These models are based on situations regularities, inducing short-term predictions about the occurrence of the onset signal. Dubois [2] defined this local, model-based anticipation as "weak anticipation".
This kind of anticipatory processes seems however unable to explain the synchronization between an agent and a more complex environment, i.e. the synchronization with an environment containing a complex structure. Dubois [2] suggests in that case the presence of a strong anticipatory process, based not on a local scale but rather on a global coordination of the entire system (agent + environment) on multiple time scales. Strong anticipation is a consequence of the coupling between the agent and the environment rather than predictions of the environment's states by the model constructed by the agent [3].

Moreover, it was suggested that the presence of such a strong anticipatory process could be revealed by a matching between the long-range correlations in the behavior of the organism and the long-range correlations in the behavior of the environment [4]. For instance in a tapping task in synchronization with a chaotic metronome, a high correlation between the fractal exponents of inter-onset intervals of the metronome and the fractal exponents of inter-tap intervals was detected [4]. This result implies that even when the environment contains no regularities the agent is sensitive to the structure of the environment. Stephen and Dixon [5] also showed, using multi-fractal analysis, that this matching of fractal exponents was not the trivial result of the sensitivity to temporal structure in aggregate but rather a subtle tendency to coordination across multiple time scales.

\subsection{Personal contribution}

The main goal of this experiment was to verify if the strong anticipation occurs in interpersonal coordination tasks. Indeed, self-paced rhythmic activities are well-known to exhibit long-range correlations[6, 7]. The presence of long-range correlations - or $1 / \mathrm{f}$ scaling - can be considered as a hallmark of complexity [8,9]. Thus when two participants are synchronized in a rhythmic activity one can consider that each of them is synchronized

This is an Open Access article distributed under the terms of the Creative Commons Attribution-Noncommercial License 3.0, which permits unrestricted use, distribution, and reproduction in any noncommercial medium, provided the original work is properly cited. 
with (and form) a complex system. We make the assumption that in an interpersonal coordination task the structure of fluctuations of the time series of periods produced by the participants within a dyad should be correlated. In order to determine whether this correlation is due to weak or strong anticipation, two kinds of analyses were conducted.

The task was performed under three conditions of coupling strength (weak, intermediate and strong): for weak anticipation higher correlation of fractal exponents with stronger coupling strength is expected, because more information about the partner behavior is available for the construction of an internal model. In contrast for strong anticipation a high correlation is expected independently of the coupling strength, from the moment where synchronization occurs. A second way for testing the nature of coordination is to analyze synchronization on short-time scales. Thus local cross-correlations (lag 0 and lag 1) analyses were conducted: for weak anticipation high cross-correlations are expected, revealing short-term adaptations. More precisely, significant cross-correlations at lag 0 are supposed to reveal the presence of internal model based on situation regularities, and significant crosscorrelations at lag 1 are supposed to reveal some reactive processes to the partner behavior. In contrast for strong anticipation significant local crosscorrelations are not expected, revealing the global matching of complexities rather than localized, shortterm adaptations.

\section{Method}

22 young healthy volunteers (16 men and 6 women, mean age 24.5 years \pm 2.9 ) were randomly paired into 11 dyads. Participants were seated sideby-side between two pendulums and each participant was instructed to hand-held one pendulum in synchrony with his partner. The angle variations of pendulums were recorded with a potentiometer located at their rotation axis, with a frequency sampling of $50 \mathrm{~Hz}$

In a first session each participant was instructed to oscillate his pendulum alone at a self-paced frequency. In a second session both participants were instructed to oscillate in synchrony at the preferred frequency of the dyad. Three trials were performed, corresponding to different levels of coupling strength. Coupling strength was modulated through the relative availability of perceptive information between participants. Each condition lasted 12 minutes, the necessary duration to collect 512 data points, which is the minimal to realize correct fractal analyzes [10].
Fractal exponents were estimated with the Detrended Fluctuation Analysis (DFA). This method is based on the analysis of the relationship between the mean magnitude of fluctuations in the series $(F(n))$ and the length of the intervals over which these fluctuations are observed $(n) . F(n)$ is linked to $n$ by a power-law:

$$
F(n) \propto n^{\alpha}
$$

In biological systems values of $\alpha$ ranging between 0.75 and 1.25 are well accepted for attesting the presence of $1 / f$ scaling [11].

The local cross-correlation analysis consists in computing cross-correlation between the $n$ first points of the series produced by the two members of a dyad. The window is then lagged by one point, and cross-correlation is computed again. This process is repeated over the whole series, yielding a series of $N$ - $n$ cross-correlation coefficients. In order to focus on short-range dependence, we worked on windows of very short length $(n=15)$.

In order to detect an effect of coupling strength on mean fractal exponents or cross-correlations, we applied one-way ANOVAs. Scheffé post-hoc tests were conducted in order to localize significant effects.

\section{Results}

The mean of fractal exponents and coefficients of correlation between fractal exponents for each condition are reported in Table 1.

The correlation between the fractal exponents of the two participants of each dyad was not significant in the non-coupling condition. In contrast, this correlation was close to one in all coupling conditions (Figure 1).

Lag 0 cross-correlations were significant in only $9.20 \%$ ), of the analyzed windows, suggesting that in more than $90 \%$ of the windows, the two series appeared independent. There was no difference between the four conditions $(\mathrm{F}(3,30)=2.29$, $\mathrm{p}=$ 0.099).

Lag 1 windowed cross-correlations were significant in $22 \%$ of the analyzed windows, suggesting the occurrence of short-term dependent epochs. The percentage of significant windows was significantly different among the four conditions $(\mathrm{F}(3,30)=4.15, \mathrm{p}=0.014)$, and the Scheffé test showed that the percentage of significant crosscorrelations was lower in the intermediate coupling condition than in the three other conditions.

\section{Discussion}



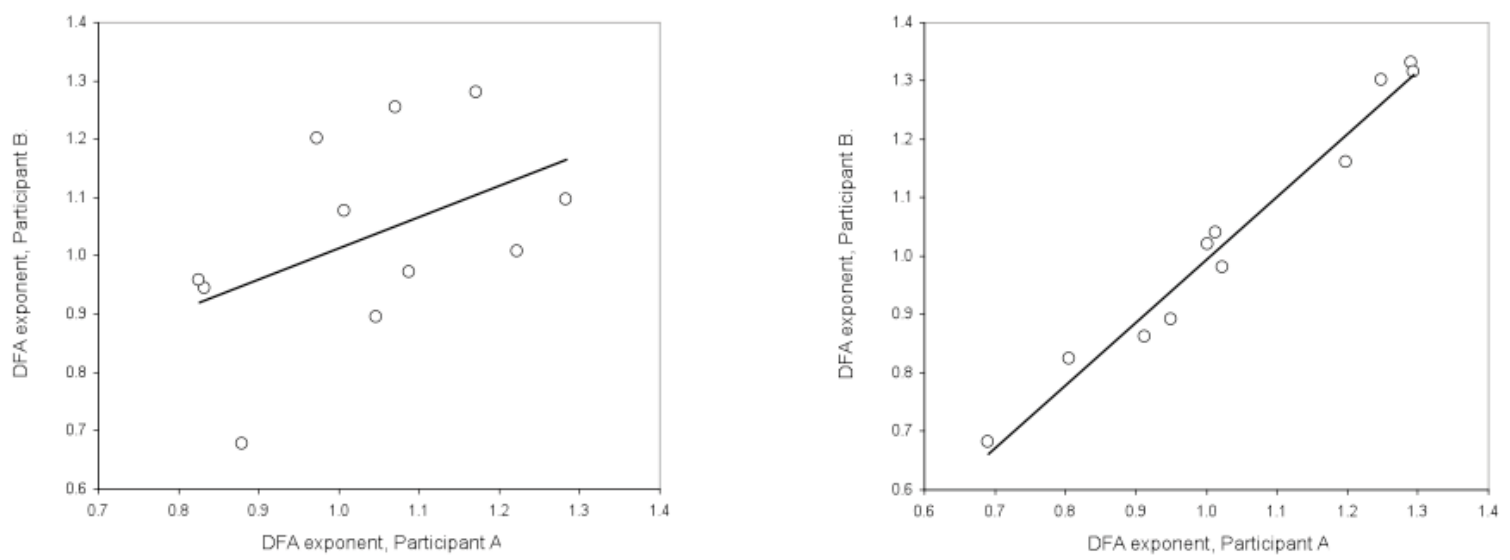

Figure 1: Correlations between DFA exponents (left: no coupling, $r=0.47$, right, strong coupling, $r=0.99$ )

The main result of this study was the very high correlation between the fractal exponents characterizing the series of periods produced by the two members of a dyad, from the moment where they acted in coordination. This very high correlation suggests a close matching of the scaling properties of the produced series. Importantly, this correlation occurred in all coupling conditions, and was independent of the strength of coupling. As previously explained, this independence between fractality matching and coupling strength favours the hypothesis of a strong form of anticipation.

Further cross-correlationnal analyses confirm that this matching of fractal exponents could not be entirely explained by short-term dependence between the series. The presence of significant lag- 0 crosscorrelation remained modest, and was not different to that observed by chance in the no-coupling condition.

The windowed analysis of lag1 cross-correlation showed that coupling tends to induce an increase of the occurrence of positive cross-correlation, indicating that one participant of the dyad tends to adjust the period produced at time $t$, in order to mimic that produced at time $\mathrm{t}-1$ by the other participant. However this occurrence of short-term dependent epochs cannot per se explain the close matching of fractal exponents: considering the three coupling conditions, there was no significant crosscorrelation in $72.2 \%$ of the analyzed windows. As a consequence, the matching of the structures of fluctuations cannot be reduced to local interactions but reveals a more global adaptation between the two complex systems.

These results support the hypothesis that the synchronization of an organism with a complex environment is mostly due to a strong anticipation process $[4,5]$. However the boundaries between weak and strong anticipation are not so clear, and probably both are acting for the synchrony with simple or complex environment, as evidenced by the

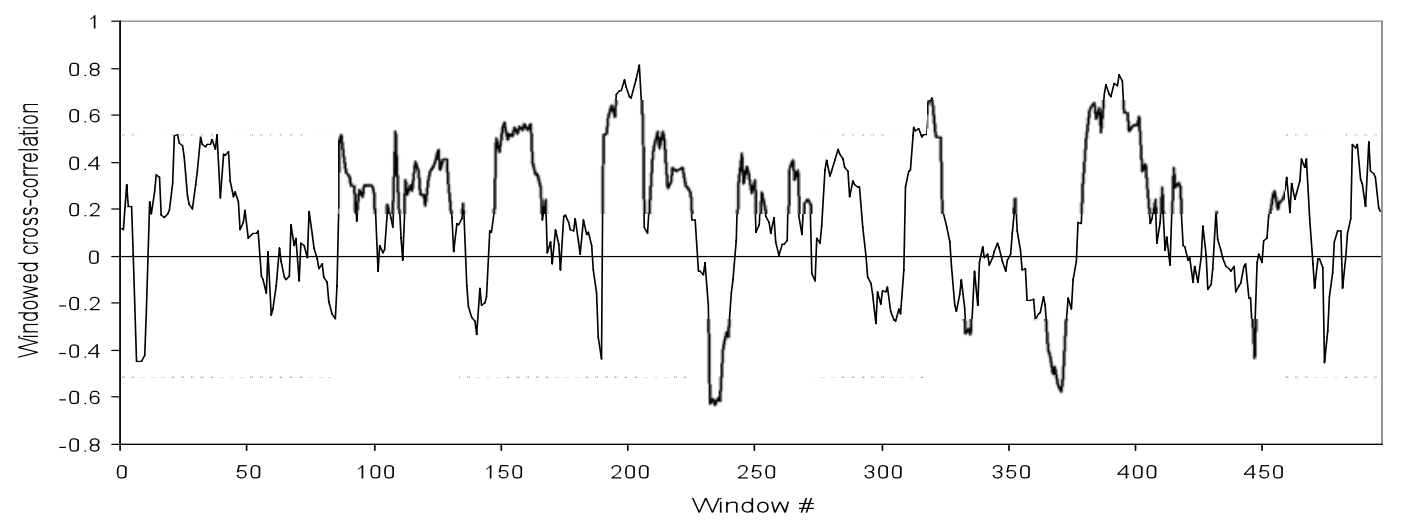

Figure 2: An example of windowed cross-correlation series (lag1, dyad \#4, strong coupling condition). The dashed lines represent the positive and negative thresholds of significance $(+/-0.5139)$. 
Table 1: Averaged results of time series analysis (standard deviation in italics): mean $\alpha$-DFA exponents and mean of correlation between the exponents of the two participants of each dyad.

\begin{tabular}{lcccc}
\hline & No coupling & $\begin{array}{c}\text { Weak } \\
\text { coupling }\end{array}$ & $\begin{array}{c}\text { Intermediate } \\
\text { coupling }\end{array}$ & $\begin{array}{c}\text { Strong } \\
\text { coupling }\end{array}$ \\
\hline$\alpha$-DFA & 1.03 & 1.03 & 0.99 & 1.04 \\
& 0.16 & 0.15 & 0.14 & 0.20 \\
Correlation/ $\alpha$-DFA & 0.47 & $0.97^{* * *}$ & $0.97^{* * *}$ & $0.99^{* * *}$ \\
\hline
\end{tabular}

$* * *: p<0.001$

presence of a low but significant percentage of local cross-correlations of periods at lag 1 . It remains a challenge to discriminate exactly the properties of such anticipatory processes, and future works should be done in this way.

These results could also open new perspectives about the "re-complexification" of organisms presenting a loss of complexity. Indeed $1 / f$ scaling is interpreted as the best compromise between order and disorder, a hallmark of adaptive and healthy systems, as it has been discovered in many young healthy systems [12, 13, 14]. Aging and some diseases are in contrast linked to a decrease of the fractal properties, time series deviating from $1 / f$ scaling either towards order (towords Brownian motion) or towards disorder (white noise). Lipsitz and Goldberger [15] defined this phenomenon as the theory of the loss of complexity with aging and disease.

A perspective should be to check whether complexity matching occurs when participants are instructed to synchronize with a metronome presenting a fractal structure in inter-onset intervals. This perspective could also join the field of virtual reality, as it could be possible to induce synchronization with a virtual environment, like an avatar, containing some fractal properties.

\section{References}

[1] B.H. Repp. Sensorimotor synchronization : A review of the tapping literature, Psychonomic Bulletin \& Review, 12 (6), 969-992, 2005.

[2] D.M. Dubois. Mathematical foundations of discrete and functional systems with strong and weak anticipations, Lecture Notes in Computer Science, 2684, 110-132, 2003.

[3] N. Stepp, and M.T. Turvey. On strong anticipation. Cognitive Systems Research, 11 (2), 148-164, 2010.

[4] D.G. Stephen, N. Stepp, J. Dixon, and M.T. Turvey. Strong anticipation: Sensitivity to long-range correlations in synchronization behavior, Physica A, 387, 5271-5278, 2008.

[5] D.G. Stephen, and J.A. Dixon. Strong anticipation: Multifractal cascade dynamics modulate scaling in synchronization behavior, Chaos, Solitons \& Fractals, 44,160-168, 2010.

[6] K. Torre, R. Balasubramaniam, and D. Delignières. Oscillating in Synchrony with a Metronome: Serial Dependence, Limit Cycle Dynamics, and Modeling, Motor Control, 14, 323-343, 2010.

[7] D. Delignières, K. Torre, and L. Lemoine. Fractal models for event- based and dynamical timers, Acta Psychologica, 127, 382-397, 2008.

[8] G.C. Van Orden, J.G. Holden, and M.T. Turvey. Selforganization of cognitive performance, Journal of Experimental Psychology: General, 132, 331-350, 2003.

[9] C.T. Kello, B.C. Beltz, J.G. Holden, and G.C. Van Orden. The emergent coordination of cognitive function, Journal of Experimental Psychology: General, 136, 551-568, 2007.

[10] D. Delignières, S. Ramdani, L. Lemoine, K. Torre, M. Fortes, and G. Ninot. Fractal analysis for short time series: a reassessment of classical methods. Journal of Mathematical Psychology, 50, 525-544, 2006.

[11] E.-J. Wagenmakers, S. Farrell, and R. Ratcliff. Estimation and interpretation of $1 / \mathrm{f}$ noise in human cognition, Psychonomic Bulletin \& Review, 11, 579$615,2004$.

[12] J.M. Hausdorff, C.K. Peng, Z. Ladin, J.Y. Wei, and A.R. Goldberger. Is walking a random walk? Evidence for long-range correlations in stride interval of human gait. Journal of Applied Physiology, 78, 349-358, 1995.

[13] C.-K. Peng, S. Havlin, H.E. Stanley, and A.L. Goldberger. Quantification of scaling exponents and crossover phenomena in non stationary heartbeat time series. Chaos, 5, 82-87, 1995.

[14] A.L. Goldberger, L.A.N. Amaral, J.M. Hausdorff, P.Ch. Ivanov, C.-K. Peng, and H.E. Stanley. Fractal dynamics in physiology: Alterations with disease and aging. Proc. Natl. Acad. Sci. USA, 99, 2466-2472, 2002.

[15] L.A. Lipsitz, and A.L. Goldberger. Loss of 'complexity' and aging: potential applications of fractals and chaos theory to senescence, JAMA, 267, $1806-1809,1992$. 